\title{
Plasma irisin depletion under energy restriction is associated with improvements in lipid profile in metabolic syndrome patients.
}

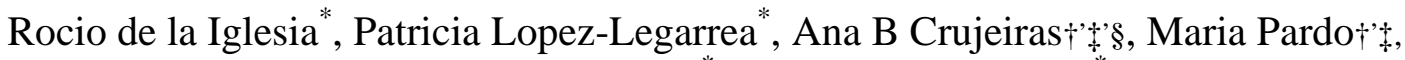
Felipe F. Casanueva†, $\ddagger$, M. Angeles Zulet ${ }^{*} \dagger$, J. Alfredo Martinez ${ }^{*} \dagger$.

*Department of Nutrition, Food Science and Physiology, University of Navarra, Pamplona, Spain, †CIBERobn Centro de Investigación Biomédica en Red, Fisiopatología de Obesidad y Nutrición, $\ddagger$ Laboratory of Molecular and Cellular Endocrinology, Instituto de Investigación Sanitaria (IDIS), Complejo Hospitalario Universitario de Santiago (CHUS) and Santiago de Compostela University (USC), Santiago de Compostela, Spain, §Cancer Epigenetics and Biology Program (PEBC), Bellvitge Biomedical Research Institute (IDIBELL), Barcelona, Spain.

\section{Correspondence:}

Name: Professor J. Alfredo Martinez

Full postal address: Department of Nutrition, Food Sciences and Physiology, University of Navarra, C/Irunlarrea, s/n, 31008, Pamplona, Navarra (Spain).

Fax number: +34 948425740

Email address: jalfmtz@unav.es 


\section{Summary}

Objective A recently discovered myokine, irisin, may have an important role in energy metabolism. This study aimed to evaluate the relationship between this hormone and the lipid profile of Metabolic Syndrome (MetS) patients following a hypocaloric diet.

Design Ninety-three Caucasian adults (52 men/41 women) diagnosed with MetS followed an 8-week-long energy restricted programme $(-30 \%$ of the energy requirements). Anthropometric measurements, biochemical markers and plasma irisin levels were analysed before and after the nutritional intervention.

Results Global plasma irisin levels were significantly reduced at the end of the study $(-72 \cdot 0 \pm 100 \cdot 9 \mathrm{ng} / \mathrm{mL}, \mathrm{p}<0 \cdot 001)$ accompanying the weight loss (-6.9\%). The depletion of irisin significantly correlated with changes in some atherogenic-related variables: total cholesterol $(\mathrm{B}=0 \cdot 106, \mathrm{p}=0 \cdot 018)$, total cholesterol/high density lipoproteincholesterol ratio ( $\mathrm{B}=0 \cdot 002, \mathrm{p}=0 \cdot 036)$, low density lipoprotein-cholesterol $(\mathrm{B}=0 \cdot 085$, $\mathrm{p}=0 \cdot 037)$ and apolipoprotein $\mathrm{B}(\mathrm{B}=0 \cdot 052, \mathrm{p}=0 \cdot 002)$, independently of changes in body weight.

Conclusions An association between the reduction of plasma irisin levels and the depletion of important lipid metabolism biomarkers was observed in patients with MetS undergoing an energy restricted programme.

Keywords: RESMENA, hypocaloric, atherogenic, cholesterol, apolipoprotein B. 


\section{Introduction}

Irisin is a recently discovered myokine, proteolytically processed from the product of the fibronectin type 3-domain containing protein 5 (FNDC5) gene ${ }^{1}$, with a possible role in the regulation of energy metabolism. As a novel hormone, several studies are being carried out in order to understand its different functions. Irisin has been reported to be expressed in skeletal muscle and to participate in white fat browning ${ }^{2}$. It might also have an antidiabetic role by improving glucose homeostasis ${ }^{3}$. Also, an inverse association with liver fat content has been described $^{4}$ and as irisin increases with exercise $^{2,5}$, a relation with follistatin, a peptide that regulates muscle growth, has been also shown ${ }^{6}$. Furthermore, an association between irisin and the lipid profile has also been described. However, results from the different studies on the target do not reach an agreement. This situation may be due to the fact that the studies have been carried out in different populations under different conditions.

In this context, the increasing prevalence of metabolic syndrome (MetS) is considered a major concern for public health authorities; they regard MetS as an important burden with relevant adverse features and complications ${ }^{7}$. Among the different metabolic abnormalities comprising the MetS, excessive adiposity and dyslipidemia have been considered of major clinical relevance due to the well-established relations with diabetes and coronary events ${ }^{8,9}$. Hence, much scientific effort is currently being directed to detect, treat and prevent MetS manifestations, focusing on decreasing body weight $^{10,11}$ and lowering the associated risks of lipid metabolism abnormalities ${ }^{12}$. In light of this, the present study aimed to investigate the possible relationships of this novel myokine (irisin) with the lipid profile of patients suffering MetS under a caloricrestricted programme: the RESMENA-S (Metabolic Syndrome Reduction in NavarraSpain) project (www.clinicaltrials.gov; NCT01087086). 


\section{Materials and methods}

\section{Study design}

This research reports the findings of a sub-analysis of the first 8-weeks of a nutritional intervention within the RESMENA-S project, which has been previously described in detail $^{13,14}$. Briefly, the study was a randomised, controlled trial that compared the effects of two hypocaloric (- 30\% of the studied requirements) dietary strategies, control and RESMENA diets, on MetS, described elsewhere ${ }^{14,15}$. The control diet was based on the American Heart Association guidelines ${ }^{16}$, including 3-5 meals per day, a macronutrient distribution of 55\% total caloric value (TCV) from carbohydrates, $15 \%$ from proteins and 30\% from lipids, as well as a healthy fatty acid (FA) profile and a cholesterol consumption below $300 \mathrm{mg}$ /day. The RESMENA diet was characterised by a higher meal frequency, consisting of seven meals per day and having a different macronutrient distribution which was as follows: $40 \%$ TCV from carbohydrates, $30 \%$ from proteins and 30\% from lipids.

Among 109 volunteers with MetS symptoms enrolled in the RESMENA-S study, a total of ninety-three Caucasian adults (52 men/ 41 women) diagnosed with MetS according to the International Diabetes Federation criteria, started the intervention. Eighty-four completed the 8-week intervention and were included in the final statistical analysis (figure 1). Participants were asked to maintain their usual physical activity, which was controlled by a 24-h physical activity questionnaire both at the beginning and at the end of the study.

This study followed the CONSORT 2010 criteria $^{18}$, and was conducted according to the guidelines laid down in the Declaration of Helsinki. Informed written consent was obtained from all the volunteers before starting the intervention trial 
(www.clinicaltrials.gov; NCT01087086) and the study was approved by the Research Ethics Committee of the University of Navarra (ref. 065/2009).

\section{Anthropometric and biochemical measurements}

Anthropometric and body composition measurements were taken in fasting conditions as was previously described ${ }^{14,19}$. Body weight was assessed to the nearest $0 \cdot 1 \mathrm{~kg}$ using bio-impedance (TANITA SC-330; Tanita Corporation) equipment. BMI was calculated as the body weight divided by height squared $\left(\mathrm{kg} / \mathrm{m}^{2}\right)$. Waist and hip circumferences were measured using a commercial measuring tape and following validated protocols ${ }^{14}$. Total body fat mass and android fat mass were determined using dual-energy X-ray absorptiometry (Lunar iDXAe, software version 6.0; GE Healthcare). Systolic blood pressure (SBP) and diastolic blood pressure (DBP) were assessed using a digital monitor (Medisana AG, MTC) according to the World Health Organization criteria. Serum total cholesterol (TC), high density lipoprotein-cholesterol (HDL-c), triglycerides (TG), free fatty acids (FFA) and glucose concentrations were measured using a Pentra C-200 auto-analyser (Horiba ABX) with specific kits. Low density lipoprotein-cholesterol (LDL-c) levels were calculated using the Friedewald formula ${ }^{20}$ : LDL-c $=$ TC - HDL-c - TG/5. Apolipoprotein B (Apo B) values were measured with a specific kit (Tina-quant Apolipoprotein B version 2) using a Model 904 Modular Roche/Hitachi autoanalyser (Roche Diagnostics).

The quantitative measurement of irisin in human plasma samples was performed using a commercial enzyme-linked immunosorbent assay (ELISA) kit directed against amino acids 31-143 of the FNDC5 protein according to the manufacturer's instructions (Irisin ELISA Kit EK-067-52; Phoenix Pharmaceuticals, INC, CA). Absorbance from each sample was measured in duplicate using a spectrophotometric microplate reader at wavelength of 450nm (Versamax Microplate Reader; Associates of Cape Cod 
Incorporated, East Falmouth, MA). This test provided a range of detection of 0.066$1024 \mathrm{ng} / \mathrm{mL}$ and exhibited an inter and intra-assay coefficient of variation of $6-10 \%$. The samples were kept at $-80^{\circ} \mathrm{C}$ and were analysed during the month following the end of the study.

\section{Statistical analyses}

This study is a sub-analysis of data obtained as a part of the RESMENA-S study. Statistical tests were performed using the SPSS $15 \cdot 0$ software for Windows (SPSS Ibérica, Madrid, Spain). Mean values and standard deviations of the measured variables were reported. Differences were considered statistically significant at $\mathrm{p}<0 \cdot 050$.

Based upon previous studies ${ }^{21,22}$, the required sample size (forty per group) in the randomised controlled trial was calculated to detect a difference of $4.3 \mathrm{~cm}$, with a variation of $\pm 6 \cdot 8 \mathrm{~cm}$ between the groups in the reduction of waist circumference with a p $<0.05$ and a power of $80 \%$. The estimated dropout rate was 25\%, and the initial number of recruited subjects was 109. The intervention trial started with ninety-three subjects presenting MetS and ended with forty per group (figure 1).

Differences between the beginning and the end of the intervention were analysed in each dietary group by a paired t-test. Differences in changes between the dietary groups were assessed by analyses of covariance after adjusting for baseline values of each variable. One-tailed Pearson correlation analysis was used to test for a positive relationship between percentage of depletion of irisin levels and percentage of body weight reduction with regard to baseline values. Pearson correlation analyses were performed in order to assess the potential relationships and associations among the changes in plasma irisin levels and co-variates of interest. Finally, linear regression analyses, adjusted for body weight variation, were applied to individually study associations between the changes in plasma irisin levels and variations in lipid profile 
variables (TC, HDL-c, LDL-c, TC/HDL-c ratio, Apo B, TG and FFA).

\section{Results}

\section{Anthropometric and biochemical markers}

Both dietary strategies proved to be effective in improving anthropometric and biochemical markers (table 1). Overall, control and RESMENA groups reduced body weight, BMI, waist circumference, waist to hip ratio (WHR) as well as total and android fat mass percentages with a statistically significant $\mathrm{p}$ value $<0 \cdot 001$. Both dietary strategies also resulted in a decline in the SBP and DBP measurements. As for biochemical markers, Apo B, TG and glucose levels were significantly diminished in both dietary groups. TC, HDL-c and LDL-c reductions only reached statistical significance in the control subjects, although they decreased in both dietary groups. Indeed, no statistically significant differences between control and RESMENA dietary strategies were observed in any of the anthropometric and biochemical variables (table 1). Therefore, both dietary groups were merged for further analyses.

Regarding plasma irisin levels, they presented a statistically significant reduction in both dietary groups $(-87 \cdot 3 \pm 114 \cdot 2 \mathrm{ng} / \mathrm{mL}$ control, $-57 \cdot 4 \pm 85 \cdot 1 \mathrm{ng} / \mathrm{mL}$ RESMENA, both $\mathrm{p}<0 \cdot 001$ ), but no differences were found between either nutritional patterns (table 1) or between genders.

\section{Association between irisin and lipid profile}

In the whole sample, a positive relationship was found between the percentage of depletion of irisin levels and the percentage of body weight reduction as compared to baseline values, resulting in a trend towards significance $(r=0 \cdot 165, p=0 \cdot 069)$. Moreover, significant associations between the reduction in irisin levels and a depletion in TC $(r=0 \cdot 269, p=0 \cdot 014)$, TC/HDL-c $(r=0 \cdot 241, p=0.029)$, LDL-c $(r=0 \cdot 239, p=$ 
0.031) and Apo B ( $r=0 \cdot 345, \mathrm{p}=0.002)$ were observed. However, changes in total lean mass, SBP, DBP, HDL-c, TG, FFA, glucose or activity level did not show a significant correlation with the irisin variation (table 2).

Linear regression analyses, adjusted for body weight variation, were applied to evaluate the relationship between changes in plasma irisin levels $(\mathrm{ng} / \mathrm{mL})$ and lipid profile biomarker variation (mg/dL), independently of changes in body weight. Among these, changes in TC $\left(B=0 \cdot 106\right.$, CI95\%: 0.019 to 0·193, $\left.p=0 \cdot 018, r^{2}=0 \cdot 105\right)$, TC/HDL-C ratio $\left(B=0 \cdot 002\right.$, CI95\%: 0.001 to 0.004, $\left.p=0 \cdot 036, r^{2}=-0 \cdot 100\right)$, LDL-C $(B=0 \cdot 085$, CI95\%: $0 \cdot 005$ to $\left.0 \cdot 165, \mathrm{p}=0 \cdot 037, \mathrm{r}^{2}=0 \cdot 050\right)$ and Apo B (B $=0 \cdot 052$, CI95\%: 0.020 to $0 \cdot 084, p=0 \cdot 002, r^{2}=0 \cdot 107$ ) were statistically related to plasma irisin variations (figure 2). However, for HDL-c $(p=0 \cdot 978)$, TG $(p=0 \cdot 204)$ or FFA $(p=0 \cdot 364)$ this association was not statistically significant.

\section{Discussion}

The recently discovered myokine, irisin, has received much attention due to the fact that it has been involved in the regulation of human energy metabolism ${ }^{23,24}$. In the present study it was found that the variation in atherogenic parameters paralleled the changes in irisin levels after weight loss therapy. Therefore, these results suggest that irisin could also be involved in fat metabolism homeostasis or lipid disorders.

As has been found in other studies concerning hypocaloric diets ${ }^{25}$, both control and RESMENA dietary strategies proved to be effective in improving MetS abnormalities. This conclusion is based on the fact that the general anthropometric and biochemical parameters of the volunteers that are related to MetS were lowered.

Interestingly, irisin levels decreased in both dietary groups after the 2-month-long hypocaloric intervention. Relevant investigations have reported that irisin levels are 
elevated under obesity and on MetS patients or reduced under anorexia or after weight $\operatorname{loss}^{26,27}$. In order to elucidate if the causes of the observed irisin depletion were in line with the aforementioned investigations, the association between this hormone's variation and changes in body weight, was evaluated. It is worth noting that, a trend towards significance was found in the relationship between the percentage change of irisin levels and the percentage variation of body weight.

The main objective of the current analysis was to evaluate the potential relationships between changes in atherogenic parameters and the variation in irisin levels after a weight loss programme. However, irisin changes can also be related to lean mass and/or exercise level, since it is considered a myokine ${ }^{2,5,28}$. A positive correlation between irisin and blood pressure has also been observed ${ }^{27,29}$. Moreover, it has previously been described that plasma glucose could influence serum irisin levels ${ }^{30}$. Therefore, in order to exclude possible confounding variables, correlation analyses were performed between changes in plasma irisin levels and changes in total lean mass, blood pressure, glucose concentrations or activity level. Nevertheless, no significant relationships were found in this experimental trial.

After finding irisin depletion in the MetS population at the end of the weight loss programme, the analysis of potential relationships of this myokine with the changes in the lipid profile was aimed. Associations between this myokine levels and plasma cholesterol have been previously described in other studies, but there is no general agreement among investigations concerning obese subjects. While Liu et al. ${ }^{29}$ showed a positive correlation between this myokine and TC, Huh et al. ${ }^{31}$ reported a negative association and Wen et al. ${ }^{32}$ observed a positive correlation between irisin and HDL-c, but not with TC or LDL-c. Furthermore, to our knowledge, this is the first report that shows a relationship between irisin and the atherogenic index TC/HDL-c or Apo B 
levels. Additionally, it should be mentioned that all the above cited studies were crosssectional studies, while our findings belong to a weight-loss intervention trial where an association between irisin changes and TC, LDL-c, TC/HDL-c and Apo B variations is reported.

Although the exact mechanisms of irisin action remain unclear, these findings suggest that irisin may participate in the regulation of lipid metabolism. We speculated that given irisin’s putative role as a "metabolism-activator”, changes in irisin concentration might reflect a response to metabolic (or atherogenic) burdens. This outcome might therefore explain our observation of a positive correlation between irisin and atherogenic factors such as total cholesterol and Apo B. Moreover, a resistance phenomenon could not be excluded. Indeed, irisin could follow a similar pattern as leptin or insulin, which are elevated in obese subjects and reduced after subjects undergo a hypocaloric diet ${ }^{33}$, as other authors have also suggested ${ }^{27,34}$.

In addition to the limitations of a post-hoc analysis, it should be mentioned that due to the relatively small sample size, type 2 errors cannot be excluded. Moreover, the investigation was performed on patients suffering MetS following an energy restricted diet; therefore, further studies should be carried out in order to determine whether irisin changes are related to lipid profile in other populations.

In summary, this study shows a positive relationship between the decrease in irisin levels and reductions in plasma lipid profile markers in MetS patients following an energy restricted programme. Further investigations into this myokine might be a good target to better understand not only energy expenditure, as was initially proposed ${ }^{1,2}$, but also the atherogenic dyslipidemia process, which is one of the main concerns of the MetS and obesity conditions. 


\section{Acknowledgments}

The present work was supported by the Health Department of the Government of Navarra (48/2009) and the Línea Especial about Nutrition, Obesity and Health (University of Navarra LE/97). The support from CIBERobn and RETICS schemes is gratefully accredited. The authors thank the volunteers for taking part in this study and the physician Blanca E. Martínez de Morentín, the nurse Salomé Pérez and the technician Verónica Ciaurriz for excellent technical assistance at the Metabolic Unit of the University of Navarra, as well as the technician María Amil from the Instituto de Investigación Sanitaria de Santiago de Compostela (IDIS). The authors would like to thank Carmen de la Iglesia (bachelor in Translation and Interpreting) from the Universidad Autónoma de Madrid, as well as Paul Miller, Senior lecturer in Modern Languages at the University of Navarra, for final proofreading the English language of the manuscript. Carlos III Health Institute (ISCIII) provided a predoctoral grant to R. de la Iglesia ( $\mathrm{n}^{\circ}$ FI10/00587). Government of Navarra provided a predoctoral research scholarship to Patricia Lopez-Legarrea ( $n^{\circ}$ 233/2009). A.B. Crujeiras is funded by the ISCIII through a research contract "Sara Borrell” (C09/00365) and M. Pardo is a Miguel Servet Fellow (ISCIII/SERGAS).

\section{Conflict of Interest}

The authors declare no conflict of interest. 


\section{Bibliography}

1. Bostrom, P., Wu J., Jedrychowski M.P., et al. (2012) A PGC1-alpha-dependent myokine that drives brown-fat-like development of white fat and thermogenesis. Nature, 481, 463-468.

2. Spiegelman, B.M. (2013) Regulation of adipogenesis: toward new therapeutics for metabolic disease. Diabetes, 62, 1774-1782.

3. Hojlund, K., Bostrom P. (2013) Irisin in obesity and type 2 diabetes. Journal of Diabetes and its Complications, 27, 303-304.

4. Zhang, H.J., Zhang X.F., Ma Z.M., et al. (2013) Irisin is inversely associated with intrahepatic triglyceride contents in obese adults. Journal of Hepatology, 59, 557-562.

5. Kraemer, R.R., Shockett P., Webb N.D., et al. (2013) A Transient Elevated Irisin Blood Concentration in Response to Prolonged, Moderate Aerobic Exercise in Young Men and Women. Hormone and Metabolic Research, DOI: 10.1055/s-0033-1355381.

6. Vamvini, M.T., Aronis K.N., Panagiotou G., et al. (2013) Irisin mRNA and circulating levels in relation to other myokines in healthy and morbidly obese humans. European Journal of Endocrinology, DOI: 10.1530/EJE-13-0276.

7. Lansdown, A., Ludgate M., Rees A. (2012) Metabolic syndrome: is the preadipocyte to blame? Clinical Endocrinology, 76, 19-20.

8. Araujo, A.B., Chiu G.R., Christian J.B., et al. (2013) Longitudinal changes in high-density lipoprotein cholesterol and cardiovascular events in older adults. Clinical endocrinology, DOI: 10.1111/cen.12212. 
9. Lewington, S., Whitlock G., Clarke R., et al. (2007) Blood cholesterol and vascular mortality by age, sex, and blood pressure: a meta-analysis of individual data from 61 prospective studies with 55,000 vascular deaths. Lancet, 370, 1829-1839.

10. Baxheinrich, A., Stratmann B., Lee-Barkey Y.H., et al. (2012) Effects of a rapeseed oil-enriched hypoenergetic diet with a high content of alpha-linolenic acid on body weight and cardiovascular risk profile in patients with the metabolic syndrome. British Journal of Nutrition, 108, 682-691.

11. Abete, I., Astrup A., Martinez J.A., et al. (2010) Obesity and the metabolic syndrome: role of different dietary macronutrient distribution patterns and specific nutritional components on weight loss and maintenance. Nutrition reviews, 68, 214-231.

12. Shearer, G.C., Pottala J.V., Hansen S.N., et al. (2012) Effects of prescription niacin and omega-3 fatty acids on lipids and vascular function in metabolic syndrome: a randomized controlled trial. Journal of lipid research, 53, 24292435.

13. Lopez-Legarrea, P., de la Iglesia R., Abete I., et al. (2013) Short-term role of the dietary total antioxidant capacity in two hypocaloric regimes on obese with metabolic syndrome symptoms: the RESMENA randomized controlled trial. Nutrition \& metabolism, 10, 22.

14. Zulet, M.A., Bondia-Pons I., Abete I., et al. (2011) The reduction of the metabolyc syndrome in Navarra-Spain (RESMENA-S) study: a multidisciplinary strategy based on chrononutrition and nutritional education, 
together with dietetic and psychological control. Nutricion Hospitalaria, 26, 1626.

15. de la Iglesia, R., Lopez-Legarrea P., Abete I., et al. (2013) A new dietary strategy for long-term treatment of the metabolic syndrome is compared with the American Heart Association (AHA) guidelines: the MEtabolic Syndrome REduction in NAvarra (RESMENA) project. British Journal of Nutrition, 23, 110.

16. Grundy, S.M., Cleeman J.I., Daniels S.R., et al. (2005) Diagnosis and management of the metabolic syndrome: an American Heart Association/National Heart, Lung, and Blood Institute Scientific Statement. Circulation, 112, 2735-2752.

17. Food and Nutrition Board, National Research Council (1989) Recomended Dietary Allowances: 10th edition. The National Academy Press.

18. Moher, D., Hopewell S., Schulz K.F., et al. (2012) CONSORT 2010 explanation and elaboration: updated guidelines for reporting parallel group randomised trials. International Journal of Surgery, 10, 28-55.

19. de la Iglesia, R., Lopez-Legarrea P., Celada P., et al. (2013) Beneficial Effects of the RESMENA Dietary Pattern on Oxidative Stress in Patients Suffering from Metabolic Syndrome with Hyperglycemia Are Associated to Dietary TAC and Fruit Consumption. International Journal of Molecular Sciences, 14, 69036919.

20. Friedewald, W.T., Levy R.I., Fredrickson D.S. (1972) Estimation of the concentration of low-density lipoprotein cholesterol in plasma, without use of the preparative ultracentrifuge. Clinical Chemistry, 18, 499-502. 
21. Konig, D., Deibert P., Frey I., et al. (2008) Effect of meal replacement on metabolic risk factors in overweight and obese subjects. Annals of Nutrition and Metabolism, 52, 74-78.

22. Katcher, H.I., Legro R.S., Kunselman A.R., et al. (2008) The effects of a whole grain-enriched hypocaloric diet on cardiovascular disease risk factors in men and women with metabolic syndrome. The American Journal of Clinical Nutrition, 87, 79-90.

23. Polyzos, S.A., Kountouras J., Shields K., et al. (2013) Irisin: A renaissance in metabolism? Metabolism: clinical and experimental, 62, 1037-1044.

24. Swick, A.G., Orena S., O'Connor A. (2013) Irisin levels correlate with energy expenditure in a subgroup of humans with energy expenditure greater than predicted by fat free mass. Metabolism, 62, 1070-1073.

25. Fernandez, J.M., Rosado-Alvarez D., Da Silva Grigoletto M.E., et al. (2012) Moderate-to-high-intensity training and a hypocaloric Mediterranean diet enhance endothelial progenitor cells and fitness in subjects with the metabolic syndrome. Clinical Science, 123, 361-373.

26. Stengel, A., Hofmann T., Goebel-Stengel M., et al. (2013) Circulating levels of irisin in patients with anorexia nervosa and different stages of obesity-correlation with body mass index. Peptides, 39, 125-130.

27. Park, K.H., Zaichenko L., Brinkoetter M., et al. (2013) Circulating Irisin in Relation to Insulin Resistance and the Metabolic Syndrome. The Journal of clinical endocrinology and metabolism, DOI:10.1210/jc.2013-2373. 
28. Wrann, C.D., White J.P., Salogiannnis J., et al. (2013) Exercise Induces Hippocampal BDNF through a PGC-1alpha/FNDC5 Pathway. Cell metabolism, DOI: 10.1016/j.cmet.2013.1009.1008.

29. Liu, J.J., Wong M.D., Toy W.C., et al. (2013) Lower circulating irisin is associated with type 2 diabetes mellitus. Journal of Diabetes and its Complications, 4, 365-369.

30. Choi, Y.K., Kim M.K., Bae K.H., et al. (2013) Serum irisin levels in new-onset type 2 diabetes. Diabetes Research and Clinical Practice, 100, 96-101.

31. Huh, J.Y., Panagiotou G., Mougios V., et al. (2012) FNDC5 and irisin in humans: I. Predictors of circulating concentrations in serum and plasma and II. mRNA expression and circulating concentrations in response to weight loss and exercise. Metabolism, 61, 1725-1738.

32. Wen, M.S., Wang C.Y., Lin S.L., et al. (2013) Decrease in irisin in patients with chronic kidney disease. PloS One, 8, e64025.

33. Cordero, P., Campion J., Milagro F.I., et al. (2011) Leptin and TNF-alpha promoter methylation levels measured by MSP could predict the response to a low-calorie diet. Journal of Physiology and Biochemistry, 67, 463-470.

34. Moreno-Navarrete, J.M., Ortega F., Serrano M., et al. (2013) Irisin is expressed and produced by human muscle and adipose tissue in association with obesity and insulin resistance. The Journal of Clinical Endocrinology and Metabolism, 98, E769-778. 